\title{
Parameter identification of a planetary rover wheel-soil contact model via Bayesian approach
}

\author{
Alberto Gallina* \\ Department of Robotics and Mechatronics \\ AGH University of Science and Technology \\ ul. Mieckiewica 30, 30059 Krakow, Poland \\ alberto.gallina@agh.edu.pl \\ Marco Scharringhausen \\ Institute of Space System \\ German Aerospace Center (DLR) \\ Robert Hooke Str. 7, 28359 Bremen, Germany \\ marco.scharringhausen@dlr.de
}

\author{
Rainer Krenn \\ Robotics and Mechatronics Center \\ German Aerospace Center (DLR) \\ Münchner Str. 20, 82234 Weßling, Germany \\ rainer.krenn@dlr.de \\ Tadeusz Uhl \\ Department of Robotics and Mechatronics \\ AGH University of Science and Technology \\ ul. Mieckiewica 30, 30059 Krakow, Poland \\ tuhl@agh.edu.pl
}

\author{
Bernd Schäfer \\ Robotics and Mechatronics Center \\ German Aerospace Center (DLR) \\ Münchner Str. 20, 82234 Weßling, Germany \\ bernd.schaefer@dlr.de
}

\begin{abstract}
Soft soil contact models developed for planetary exploration rovers play an important role in the study of rover mobility. Nowadays, most of the existing contact models are based on Bekker theory which requires the evaluation of several soil parameters usually measured via bevameter tests. However, substantial differences existing between the plate-soil contact scenario and the wheel-soil contact scenario, along with large variability associated with the bevameter experiments, give rise to large uncertainty in the choice of the model parameter values. In this paper a Bayesian procedure is proposed to deal effectively with the presence of uncertainty. In the proposed approach model parameters are random variables with prior distributions derived from bevameter measurements. The prior distributions are then enhanced to posterior distributions through single wheel test data. At the end the procedure identifies a set of possible model parameter configurations that result in high experimentalnumerical matching.
\end{abstract}

\section{Introduction}

Past experience has shown that rover mobility on sandy soil is a key point in planetary exploration with wheeled vehicles. Indeed, due to loose soil, the rover may get stuck for a long time and, in worst cases, be unable to recover its mobility. One such famous example is connected with the NASA Mars rover Spirit, that got completely stuck after five years of very successful operations on Mars' surface. Therefore,

*Alberto Gallina had been a guest scientist at DLR, Robotics and Mechatronics Center, from June 2011 to September 2012. 
in the terramechanics community it is well accepted that a good knowledge of the wheel-soil contact interacting forces is a prerequisite for successful rover design and in-situ operations. In this context, computer simulations may represent a valid tool for predicting contact forces. At present, most of the existing contact models for sandy soils are based on Bekker theory (see e.g. (Wong, 2010; Ishigami et al., 2007; Leite et al., 2012; Krenn and Gibbesch, 2011)). This semi-empirical approach, although dating back to the 1950s (Bekker, 1956), surprisingly seems to be the modeling method still preferred for soil contact modeling. Indeed, it ensures a very good compromise between sufficient accuracy and speed of simulation runs. However, despite its large usage, several concerns arise when applying Bekker approach to wheel-soil contact models. Bekker parameters, often assessed through bevameter tests, are proved to be highly sensitive to the test setup, such as for instance soil preparation, plate shape or velocity (Apfelbeck et al., 2011). Thus, inherent variability in the testing process results in significant uncertainty about the measured parameters. Furthermore, Bekker theory is based on uniaxial pressure-sinkage and symmetric plate shear tests. Its extension to tridimensional and generally more complex wheel-soil contact geometry is not fully justified. This also gives rise to uncertainty about the correctness of the model itself.

In this paper, the Bayesian framework is employed to identify adequate model parameters in the presence of both model and parameter uncertainty. The procedure, recently proposed in (Gallina et al., 2012), is presented here with comprehensive analyses. In the Bayesian framework model parameters are considered to be random variables whose distribution, initially defined by limited information, is progressively improved as soon as new experimental evidence is made available. In the presented case study, initial parameter knowledge, given by bevameter measurements, is corrected by experimental data from single wheel tests. A non-deterministic description of the parameters gives the researcher a deep insight into the model behavior and allows him or her to make robust decision when uncertainties are considered.

The structure of the paper is as follows. First, general theoretical aspects of Bayesian model updating are given. Second, the soil contact model, whose parameters are identified via Bayesian approach, is presented. Third, the Bayesian procedure setup for the specific terramechanics problem is described. Finally, two application cases are illustrated.

\section{Theoretical fundamentals of Bayesian parameter identification procedure}

The choice of the model parameter values is one of the fundamental steps to be addressed when computer models are used to describe physical processes. The parameters should be chosen such that they are consistent with real physical values and at the same time produce results in good agreement with experimental evidence. This problem, referred to as parameter identification, is not trivial at all as only a very limited knowledge of the phenomenon under study is usually available. In a Bayesian approach the uncertainty affecting the model parameter is described by random variables. Thus, the model parameters are denoted by the vector $\boldsymbol{\theta}_{p}$, where $\boldsymbol{\theta}_{p}$ is a random vector with probability density function $p\left(\boldsymbol{\theta}_{p}\right)$. This probability distribution is called prior distribution and its shape reflects the available initial knowledge on the parameter values. Yet, in a Bayesian framework it is assumed that uncertainty occurs also in the computer model and is expressed through the model prediction error $\epsilon$ defined by

$$
\epsilon=x-y,
$$

with $y$ denoting generic model response and $x$ the corresponding experimental data. The model prediction error is also assumed to be a random variable. In the absence of information about the model error uncertainty, $\epsilon$ can be assumed to be a Gaussian random variable with probability density

$$
p(\epsilon)=\frac{1}{\sqrt{2 \pi} \sigma} \exp \left(-\frac{(\epsilon-\bar{\epsilon})^{2}}{2 \sigma^{2}}\right),
$$


where the mean prediction error $\bar{\epsilon}$ is usually considered to be equal to the numerical solution $y$ and the standard deviation $\sigma$ is fixed to a particular value or is considered an additional parameter to estimate. The choice of Gaussian distribution is very common because it maximizes the model uncertainty, according to the principle of maximum entropy (Beck and Katafygiotis, 1998). Plugging Equation (1) into Equation (2) the following conditional probability can be written

$$
p(x \mid \boldsymbol{\theta})=\frac{1}{\sqrt{2 \pi} \sigma} \exp \left(-\frac{\left[x-y\left(\boldsymbol{\theta}_{p}\right)\right]^{2}}{2 \sigma^{2}}\right),
$$

where the dependency of $y$ on the parameter vector $\boldsymbol{\theta}_{p}$ is made explicit. In Equation (3) the vector $\boldsymbol{\theta}$ represents the parameter vector extended with the prediction error standard deviation, namely $\boldsymbol{\theta}=\left[\boldsymbol{\theta}_{p}^{\prime} \sigma\right]^{\prime}$ (where the sign " denotes the transpose). Equation (3) is referred to as likelihood function and represents the probability of observing the experimental response $x$ when the corresponding numerical model has parameters $\boldsymbol{\theta}_{p}$ and prediction error standard deviation $\sigma$. In other words, the likelihood function measures the fitness of the model response to the experimental response. Using the Bayes rule it is possible to evaluate the inverse conditional probability $p(\boldsymbol{\theta} \mid x)$ as

$$
p(\boldsymbol{\theta} \mid x)=\frac{p(x \mid \boldsymbol{\theta}) p(\boldsymbol{\theta})}{p(x)} .
$$

$p(\boldsymbol{\theta} \mid x)$ is called posterior distribution and estimates the parameter distribution given the experimental data $x$. From a different perspective, the posterior distribution is the prior distribution corrected by the experimental data $x$. In Equation (4) the probability $p(x)$ is a normalizing factor that does not affect the $p(\boldsymbol{\theta} \mid x)$ shape. When a set of experimental data $D$ is given, Equation (4) can be more generally written as

$$
p(\boldsymbol{\theta} \mid D)=\frac{p(D \mid \boldsymbol{\theta}) p(\boldsymbol{\theta})}{p(D)},
$$

with the likelihood function $p(D \mid \boldsymbol{\theta})$ defined by

$$
p(D \mid \boldsymbol{\theta})=\prod_{i=1}^{N} \frac{1}{\sqrt{2 \pi} \sigma_{i}} \exp \left(-\frac{\left[x_{i}-y_{i}\left(\boldsymbol{\theta}_{p}\right)\right]^{2}}{2 \sigma_{i}^{2}}\right),
$$

where $x_{i}, y_{i}, \sigma_{i}$ denote the $i$-th experimental response, numerical response and prediction error standard deviation, respectively, with $i=1 \ldots N$ and $N$ the number of observed responses. In Equation (6) independence among the $N$ model prediction errors has been assumed. Under this assumption, the prediction error covariance matrix is diagonal with diagonal terms defining the vector $\boldsymbol{\sigma}=\left[\sigma_{1} \ldots \sigma_{N}\right]^{\prime}$. Thus, the extended parameter vector becomes now $\boldsymbol{\theta}=\left[\boldsymbol{\theta}_{p}^{\prime} \boldsymbol{\sigma}^{\prime}\right]^{\prime}$.

The evaluation of the posterior distribution is called inference problem and represents the goal of a parameter identification problem. The inference problem is challenging because the distribution of $p(D \mid \boldsymbol{\theta}) p(\boldsymbol{\theta})$ is usually very complex and because the normalizing factor is a multidimensional integral that may not be solved in practice, either analytically or numerically. In literature, three different approaches have been presented to make inference: exact methods, deterministic approximation, stochastic approximation (Bishop, 2006). Even though the first two methods have several advantages in terms of computational burden, they are not universal and sometimes difficult to apply. Conversely, stochastic approximation methods, based on sampling strategies, are simple, universal and ensure asymptotic convergence to the right solution. In this paper a particular procedure, which belongs to the class of Markov Chain Monte Carlo (MCMC) methods (Metropolis et al., 1953), is employed to calculate the posterior distribution. Markov Chain Monte Carlo is a powerful technique for efficient sampling from complex probability distribution that is widely used to make inference. Limitations of the conventional Metropolis-Hasting MCMC algorithm in Bayesian parameter identification are due to the fact that $p(\boldsymbol{\theta} \mid D)$ is usually very peaked. Therefore, an MCMC approach will be effective as long as a large number of samples is chosen in the neighborhood of the important (but unknown) region of the parameter space, that is the region where the likelihood function is high. In an attempt to alleviate this problem, Transitional Markov Chain Monte Carlo (TMCMC) algorithm has been recently proposed (Ching 
and Chen, 2007). TMCMC entails a sequence of Bayes' problems where the posterior distribution of the previous step is used as the prior distribution of the current step and the amount of exploited experimental data is gradually increased. This sequential approach favors the movement of the posterior distribution toward highly important regions of the parameter space. TMCMC has been already successfully applied to Bayesian updating (Ching and Chen, 2007; Goller et al., 2011) and model classification problems (Muto, 2007), and it is proposed here in the context of soil contact model parameter identification. Theoretical aspects of TMCMC can be found in (Ching and Chen, 2007).

\section{Wheel-soil contact model}

The wheel-soil interaction dynamics implementation is based on the soil contact model (SCM) (Krenn and Gibbesch, 2011), which is dedicated to multi-body dynamics simulations. It computes the contact forces and torques between an arbitrarily shaped contact body and a soft terrain surface, as function of the body's motion state. The parameters of SCM can be divided in two categories: the first category of parameters describes the surface shape geometries of the contact body (here a wheel) and of the terrain in form of a CAD-like surface mesh for the contact body and of a digital elevation model (DEM) of the terrain. The second category defines the dynamical soil properties that are to be identified by the Bayesian procedure. A list of the soil parameters used in SCM is given in Table 1.

\begin{tabular}{lll}
\hline Parameter & Variable & Unit \\
\hline Exponent of sinkage & $n$ & {$[-]$} \\
Cohesive modulus & $k_{c}$ & {$\left[\mathrm{~N} / \mathrm{m}^{n+1}\right]$} \\
Frictional modulus & $k_{\phi}$ & {$\left[\mathrm{N} / \mathrm{m}^{n+2}\right]$} \\
Cohesion & $c$ & {$[\mathrm{~Pa}]$} \\
Angle of internal friction & $\Phi$ & {$[\mathrm{rad}]$} \\
Areal damping coefficient of soil & $D_{\text {soil }}$ & {$\left[\mathrm{Ns} / \mathrm{m}^{3}\right]$} \\
Velocity specific Janosi coefficient & $J$ & {$[\mathrm{~m} / \mathrm{s}]$} \\
\hline
\end{tabular}

Table 1: Soil parameters used in SCM.

\subsection{Contact dynamics computation}

The first computational task of SCM is detecting contact between the moving contact body and the terrain. This is solved using z-buffer and spatial binning techniques known from graphics renderers. Here, the mesh grid nodes of the contact body are mapped onto the regularly spaced grid nodes of the terrain's DEM and the elevation coordinates of the mapped nodes are compared with the corresponding ones of the terrain regarding penetration. In case of contact, one obtains a footprint profile in the soil, which is a subset of the terrain DEM including all contact nodes. The elevation differences between the latest terrain surface shape and the footprint profile define the local sinkages $z_{i}$ at each contact node $i$ with location $\left(x_{i}, y_{i}\right)$. The corresponding contact velocity $\mathbf{v}_{i}$, with components $\mathbf{v}_{\sigma, i}$ normal to the footprint surface and $\mathbf{v}_{\tau, i}$ tangential to it, are computed by basic kinematics relationships for each contact node.

In the second step, SCM computes two additional variables that depend on the footprint shape: the effective contact width and the pressure distribution in the contact zone. The effective contact width is calculated by $b_{\text {eff }}=2 A_{x y} / L_{x y}$, where $A_{x y}$ is the footprint area and $L_{x y}$ the footprint contour length projected onto the horizontal $x y$-plane of the DEM. Using the variables listed in Table 1 SCM computes the normal stress $\sigma_{i}$ and the shear stress $\tau_{i}$ at all contact nodes via the equations given below that represent an extension of the well-known Bekker's equations (Bekker, 1969)

$$
\begin{gathered}
\sigma_{i}=\gamma_{i}\left(\frac{k_{c}}{b_{e f f}}+k_{\phi}\right) z_{i}^{n}+D_{s o i l} v_{\sigma, i} \\
\tau_{i}=\left(c+\sigma_{i} \tan \phi\right)\left(1-\exp ^{-\left|\frac{v_{\tau, i}}{J}\right|}\right),
\end{gathered}
$$


where $\gamma_{i}$ is a scaling factor that amplifies the soil pressure in central footprint regions and lets it drop down to zero at the footprint border. In addition to classical approaches, SCM also takes velocity dependent terms into account. For normal stress calculation (Equation (7)) a linear damping component with areal damping coefficient $D_{\text {soil }}$ is included. The inclusion of damping into the shear stress calculation (Equation (8)) is given by an exponential function derived from Janosi and Hanamoto (Janosi and Hanamoto, 1961). However, in SCM the original shear deformation term is replaced by a shear velocity term. Therefore, the so-called Janosi coefficient $J$ becomes here a reference velocity. Once the shear and normal stresses are computed, the discrete contact force $\Delta \mathbf{F}_{i}$ can be calculated by

$$
\Delta \mathbf{F}_{i}=\left(\sigma_{i} \mathbf{n}_{i}+\tau_{i} \mathbf{t}_{i}\right) \Delta A,
$$

with $\mathbf{t}_{i}=-\mathbf{v}_{\tau, i} /\left|\mathbf{v}_{\tau, i}\right|$ denoting the local tangent vector, $\mathbf{n}_{i}$ the local normal vector and $\Delta A$ the DEM grid size. Finally, the total contact force $\mathbf{F}$ and torque $\mathbf{T}$ vectors applied to the contact body are obtained by integrals of $\Delta \mathbf{F}_{i}$ over all $N$ contact nodes with

$$
\mathbf{F}=\sum_{i=1}^{N} \Delta \mathbf{F}_{i} \quad \text { and } \quad \mathbf{T}=\sum_{i=1}^{N}\left(\mathbf{r}_{i} \times \Delta \mathbf{F}_{i}\right),
$$

where $\mathbf{r}_{i}$ is the distance vector from the selected reference frame to the contact node $i$.

\subsection{Plastic soil deformation}

Typical terramechanics phenomena like sinkage, bulldozing, digging of spinning wheels or multi-pass effects of wheels rolling in line are caused by plasticity of loose soil. In order to adequately consider these effects SCM continuously updates the DEM terrain shape in each simulation step as function of the contact kinematics. In this step, SCM approximates the displacement of soil from the footprint area by defining horizontal radial and parallel soil flow fields, which are functions of the local contact velocities in normal and tangential directions. These flow fields remove soil from the footprint area and depose it around the wheel, where finally an algorithm for thermal erosion keeps the DEM surface in a natural shape considering the angle of response of the soil (additional SCM parameter not listed in Table 1). A snapshot of an animation taken from a SCM supported simulation run of a rover cruising in soft terrain is presented in Figure 1. It exemplarily shows the results of the DEM update process by means of the computed ruts of the profiled rover tires.

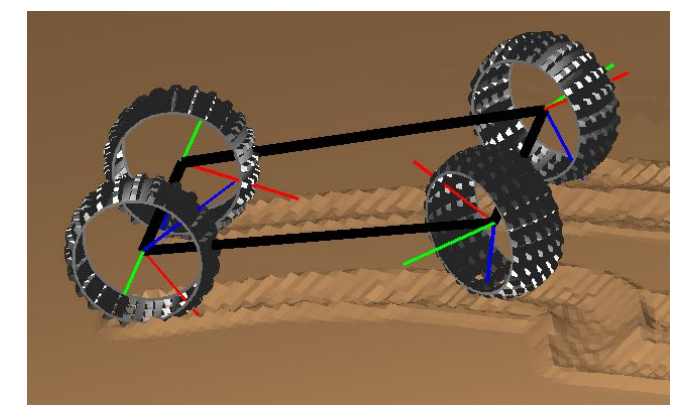

Figure 1: Visualization of rover locomotion simulation result using SCM.

\section{Procedure setup}

Bayesian approach has been used to calculate the posterior distributions of a set of SCM model parameters for future ExoMars rover mobility simulations. The procedure makes use of both single wheel test (SWT) and Bevameter tests. Bevameter tests supply the initial (prior) soil parameters assessment. The data from 
SWT, involving real wheel-soil contact scenario, allow one to improve the parameter estimates. The following subsections address important issues related to the Bayesian procedure setup for the current terramechanics problem.

\subsection{Experimental tests}

A single wheel test of a flexible wheel designed for the ESA ExoMars project was carried out at the DLR Institute of Space Systems in Bremen (Figure 2(a)). The geometric properties of the wheel include a nominal radius of $125 \mathrm{~mm}$, width of $112 \mathrm{~mm}$ and 12 straight grousers with $9 \mathrm{~mm}$ depth. During the experiment the torque, drawbar pull force and sinkage of the wheel were measured while keeping the normal force and slip ratio constant to $315 \mathrm{~N}$ and 0.2 , respectively. The wheel was driven on a dry quartz sand named WF34. The major chemical constituent of that sand was $\mathrm{SiO} 2(99.7 \%)$ composed almost exclusively of grains of sizes between 180 and $355 \mu \mathrm{m}(80 \%)$. Minor constituents were grains of sizes between 125 and $180 \mu \mathrm{m}$. The bulk density has been measured to be approximately $1400 \mathrm{~kg} / \mathrm{m}^{3}$. Bevameter tests of WF34 were previously performed to measure the soil cohesion $c \approx 0 \mathrm{~Pa}$, friction angle $\Phi \approx 30^{\circ}$, soil deformation exponent $n \approx 1.1$ and soil deformation modulus $k^{*} \approx 10^{7} \mathrm{~N} / \mathrm{m}^{\mathrm{n}+2} \cdot k^{*}$ is related to the Bekker coefficients $k_{c}$ and $k_{\phi}$ appearing in Equation (7) through

$$
k^{*}=\left(\frac{k_{c}}{b_{e f f}}+k_{\phi}\right)
$$

\subsection{Computer model}

A rigid-body model of the SWT has been created utilizing commercial software SIMPACK (Figure 2(b)). SCM is compiled in SIMAPCK as a user-defined force element acting between soil and wheel. The rigid-body model resembles the experimental SWT set-up except for the flexible wheel that is assumed to be rigid in the simulation. The influence of the elastic deformation of the real wheel is accounted for in the simulations by a wheel with increased radius. However, since the wheel deformation is not measured during tests, an equivalent radius $R$ of the rigid wheel is a parameter to be identified.

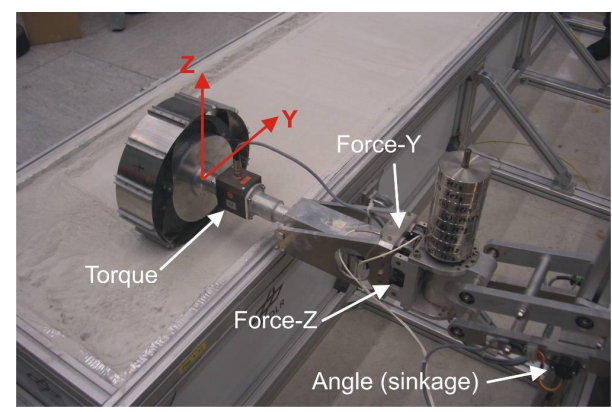

(a)

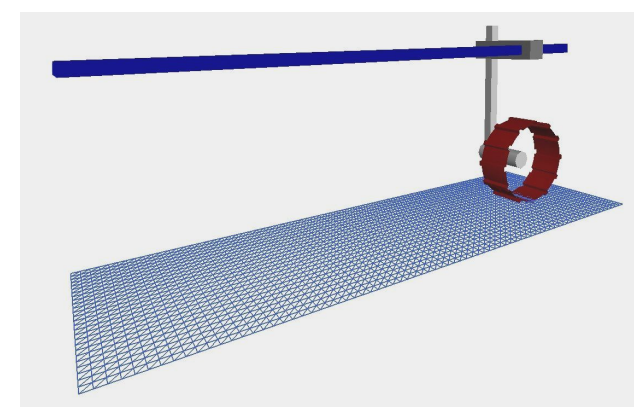

(b)

Figure 2: SWT facility (left) and rigid-body model of the single wheel test (right).

\subsection{Likelihood function}

The definition of the likelihood function represents the core of the Bayesian procedure setup. In the current analysis the likelihood is calculated by

$$
p(D \mid \boldsymbol{\theta})=\prod_{i=1}^{3} p\left(x_{i} \mid \boldsymbol{\theta}\right)
$$


with

$$
\begin{aligned}
& p\left(x_{1} \mid \boldsymbol{\theta}\right)=\frac{1}{\sqrt{2 \pi}} \exp \left(-\frac{\xi_{1}^{2}}{2}\right) \\
& p\left(x_{2} \mid \boldsymbol{\theta}\right)=\frac{1}{\sqrt{2 \pi}} \exp \left(-\frac{\xi_{2}^{2}}{2}\right) \\
& p\left(x_{3} \mid \boldsymbol{\theta}\right)=\frac{1}{\sqrt{2 \pi}} \exp \left(-\frac{\xi_{3}^{2}}{2}\right) .
\end{aligned}
$$

In Equation (13) the terms $\xi_{i}$ are standard normal variables calculated by

$$
\xi_{i}=F^{-1}\left(U_{i}\right),
$$

where $F^{-1}$ is the inverse cumulative density function of a standard normal random variable and the $U_{i}$ terms defined by

$$
\begin{aligned}
U_{1} & =\int_{-\infty}^{\bar{F}^{\text {exp }}} \frac{1}{\sqrt{2 \pi} \sigma_{F}} \exp \left(-\frac{\left(u-\bar{F}^{\text {num }}\right)^{2}}{2 \sigma_{F}^{2}}\right) d u \\
U_{2} & =\int_{-\infty}^{\bar{T}^{\text {exp }}} \frac{1}{\sqrt{2 \pi} \sigma_{T}} \exp \left(-\frac{\left(u-\bar{T}^{\text {num }}\right)^{2}}{2 \sigma_{T}^{2}}\right) d u \\
U_{3} & =\int_{-\infty}^{\bar{z}^{\text {exp }}} \frac{1}{\sqrt{2 \pi} \sigma_{z}} \exp \left(-\frac{\left(u-\bar{z}^{\text {num }}\right)^{2}}{2 \sigma_{z}^{2}}\right) d u .
\end{aligned}
$$

In Equation (15) $\boldsymbol{\bullet}^{\exp }, \boldsymbol{\bullet}^{\text {num }}$ and $\sigma_{\bullet}$ denote respectively the experimental mean, numerical mean and prediction error standard deviation of the generic physical quantity $\bullet$. The $U_{i}$ terms represent the likelihood probabilities of the considered SWT response, obtained by integrating the likelihood functions of Equation (3). The normalization step proposed above enables efficient work with data with different orders of magnitude, as in the present case for the drawbar pull force $F$ (expressed in N), the wheel torque $T$ (expressed in $\mathrm{Nm}$ ) and the wheel sinkage $z$ (expressed in $\mathrm{m}$ ). The use of average responses in the likelihood function is dictated by the fact that numerical responses, although showing the typical fluctuations caused by the grousers effect (see Figure 3), evidence poor point to point experimental-numerical correlations. Moreover, SCM is not capable of predicting the initial transient behavior exactly. Thus, the signals are averaged after an assigned offset when the steady state seems to be achieved. The offset is set to $40 \mathrm{~s}$ for $\bar{F}^{\exp }, \bar{T}^{\exp }, \bar{z}^{\exp }$, $20 \mathrm{~s}$ for $\bar{F}^{\text {num }}, \bar{T}^{\text {num }}$ and $1 \mathrm{~s}$ for $\bar{z}^{\text {num }}$. Any simulation run lasts $50 \mathrm{~s}$ while SWTs last about $180 \mathrm{~s}$. Figure 3 gives a pictorial description of the physical quantities used to define the likelihood function. The filtered experimental and numerical signals are represented by thin solid lines, while the $\bar{F}, \bar{T}$ and $\bar{z}$ values, averaged over the assigned time interval (colored background), are marked with thick solid lines. The three prediction error standard deviations $\sigma_{F}, \sigma_{T}$ and $\sigma_{z}$ appearing in Equation (15) are unknown at the beginning of the analysis and represent additional parameters to be identified.
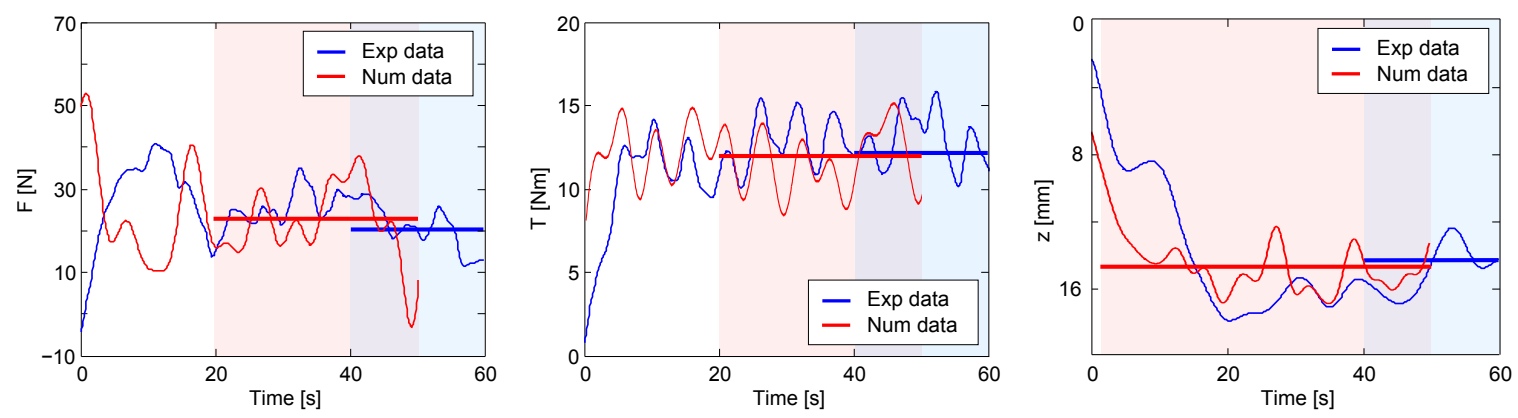

Figure 3: Filtered signals of experimental (only first $60 \mathrm{~s}$ ) data and numerical solution for a particular model configuration. 


\subsection{Sensitivity analysis}

SCM requires the definition of the 7 soil parameters listed in Table 1. In order to reduce the number of updating parameters, a global sensitivity analysis is performed. The analysis examines the influence of the 7 SCM soil parameters on the average response of the wheel sinkage, drawbar pull force and torque calculated during SIMPACK SWT simulations. Morris method (Saltelli et al., 2000) has been employed for this aim. For each parameter-response combination, the method returns two normalized indices which give insight into the amount of linear and non-linear dependency of the response on the parameter, within the parameter space defined in Table 2. Low values of both linear and non-linear indexes point out low influence of the parameter on the response. The results, presented in Figure 4, are an evidence of the small influence of the cohesion and soil damping on all considered responses $(F, T, z)$ at low slip values. Therefore, no significant loss of information is expected if these parameters are neglected in the parameter identification process and fixed to the assigned nominal values. It should be noted that the influence of $k_{c}$ and $k_{\phi}$ was jointly studied through the $k^{*}$ parameter.
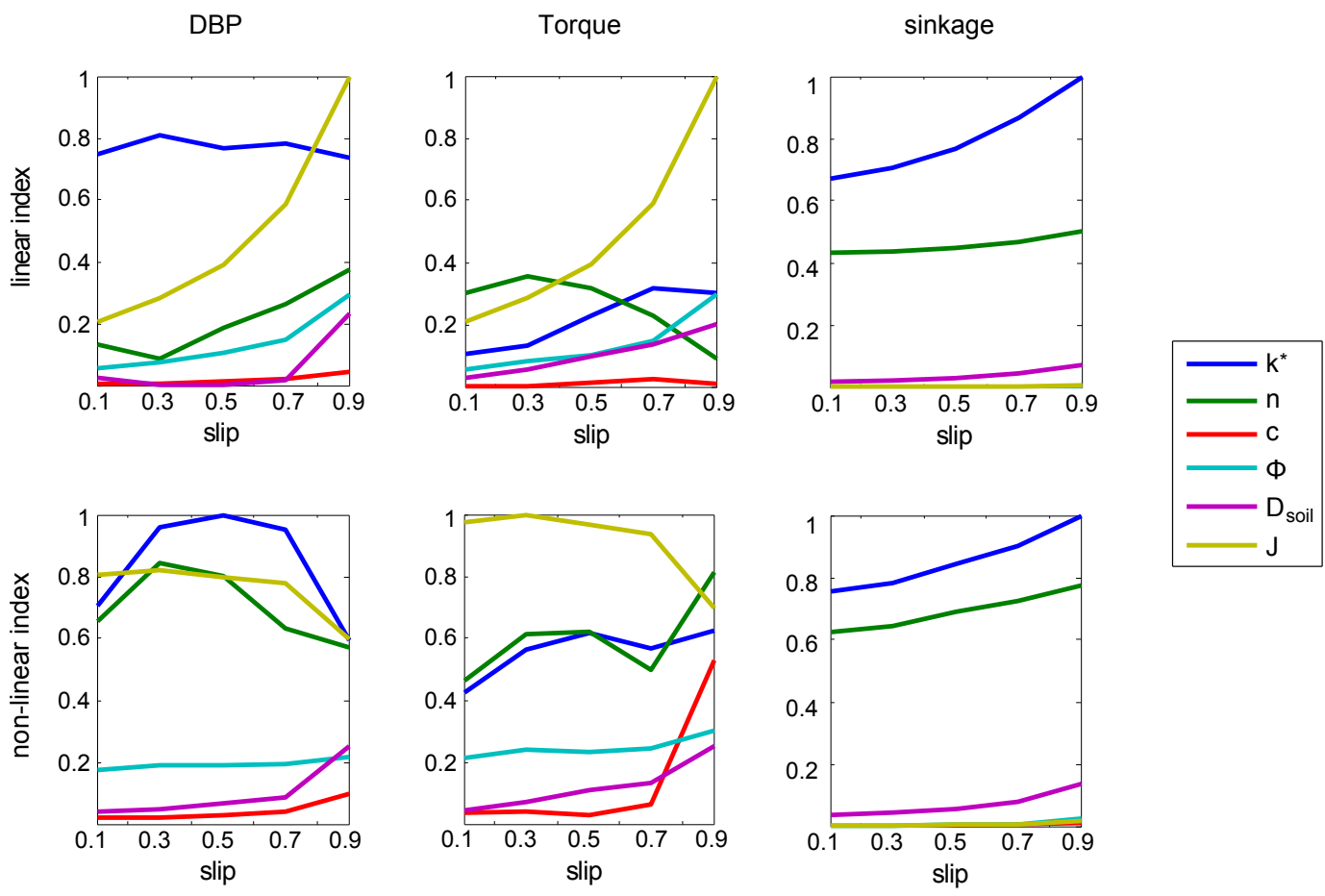

Figure 4: Linear and non-linear index of the Morris sensitivity analysis.

\subsection{Prior probabilities}

The full set of parameters to estimate is composed of $k_{c}, k_{\phi}, n, \Phi, J, R, \sigma_{F}, \sigma_{T}$ and $\sigma_{z}$. Each parameter is assumed to be an independent random variable whose prior probability density function (PDF) is assigned according to bevameter results, engineering considerations or, in the absence of any information, even mere speculation. Prior distributions of $n$ and $\Phi$ have been assumed Gaussian with mean values and standard deviations resulting form repetitive bevameter measurements. Unfortunately, the same experiments could not be employed to determine the prior distributions of $k_{c}$ and $k_{\phi}$, because they were used in the past to estimate only $k^{*}$. Thus, the prior PDFs $k_{c}$ and $k_{\phi}$ have been assumed uniform with bounds chosen according to the experience gained while working with similar soils. The distribution of the Janosi coefficient as well as the wheel radius were also considered uniform due to the fact that no information existed on more probable 
values for these parameters. Finally, the prior PDFs of the model prediction standard deviations were again uniform with the upper bounds function of the maximum expected prediction error. A summary of the prior PDFs is given in Table 2. The column of the table entitled "Nominal" defines the values used in those analyses where the parameter is not changed, "Par1" and "Par2" stand for mean and standard deviation in case of normal distribution and lower and upper bound in case of uniform distribution, while LB and UB denote the lower and upper bound of the parameter space.

\begin{tabular}{cccccccc}
\hline Param & Dim & Dist & Nominal & Par1 & Par2 & LB & UB \\
\hline$k_{c}$ & {$\left[N / m^{n+1}\right]$} & uniform & - & $-10^{9}$ & $10^{9}$ & $-10^{9}$ & $10^{9}$ \\
$k_{\phi}$ & {$\left[N / m^{n+2}\right]$} & uniform & - & 1 & $10^{10}$ & 1 & $10^{10}$ \\
$n$ & {$[-]$} & normal & - & 1.1 & 0.2 & 0.8 & 2 \\
$\Phi$ & {$\left[{ }^{\circ}\right]$} & normal & - & 30 & 5.7 & 11.5 & 37.3 \\
$J$ & {$[\mathrm{~m} / \mathrm{s}]$} & uniform & 0.001 & 0.001 & 0.1 & 0.001 & 0.1 \\
$R$ & {$[\mathrm{~m}]$} & uniform & 0.125 & 0.125 & 0.155 & 0.125 & 0.155 \\
$\sigma_{F}$ & {$[N]$} & uniform & - & 0 & 5 & 0 & $\infty$ \\
$\sigma_{T}$ & {$[N \mathrm{~m}]$} & uniform & - & 0 & 10 & 0 & $\infty$ \\
$\sigma_{z}$ & {$[\mathrm{~m}]$} & uniform & - & 0 & 0.005 & 0 & $\infty$ \\
\hline
\end{tabular}

Table 2: Prior distributions and parameter space definition.

\section{Application cases}

The analyses presented in this section are: (case 1) a TMCMC with $\boldsymbol{\theta}=\left[k_{c} k_{\phi} n \Phi J \sigma_{F} \sigma_{T} \sigma_{z}\right]^{\prime}$ and (cases $2 \mathrm{a}$ and $2 \mathrm{~b}$ ) two TMCMC repetitions with $\boldsymbol{\theta}=\left[\begin{array}{llll}k_{c} & k_{\phi} n \Phi R \sigma_{F} \sigma_{T} \sigma_{z}\end{array}\right]^{\prime}$. Each TMCMC analysis involves altogether 16000 samples split in a sequence of 2000 samples per step. The 2000 initial samples are generated according to the prior PDF while the resultant 2000 final samples describe the posterior distribution. The procedures identify parameter configurations that ensure good experimental-numerical correlation for the single wheel test responses. An example of such a correlation for a highly plausible choice of SCM parameters identified by the Bayesian procedure is depicted in Figure 3. Although the Bayesian procedure produces various results, in the following subsections we will focus only on the posterior distributions of the SCM parameters, namely $k_{c}, k_{\phi}, n, \Phi, J$ and $R$.

\subsection{Case 1}

The histograms of the prior and posterior distributions of the 5 soil parameters are drawn in Figure 5 . It is visible here how the additional knowledge provided by SWT data markedly shrinks the parameter region of plausible models with respect to the prior solution.

Bivariate contour plots of the resultant posterior distributions are also presented in Figure 6 for selected pairs of model parameters. The posterior PDFs are constructed from the posterior TMCMC samples via kernel estimation method (Bishop, 2006). These plots allow one to disclose the existing parameter correlations, as for instance the one appearing in the $k_{c^{-}} k_{\phi}$ case. Figure 6 also shows the level of agreement of the posterior PDFs with the mean values of the experimental bevameter measurements. In particular, the dashed gray line in Figure 6-left denotes the $k_{c^{-}} k_{\phi}$ combinations for which $k^{*}=10^{7} \mathrm{~N} / \mathrm{m}^{\mathrm{n}+2}$ and $b_{\text {eff }}$ is equal to the average value observed in the SWT simulations. Good correlation between the bevameter measurement and the estimated value exists for cohesion modulus, friction modulus and sinkage exponent. This means that, at least for the analyzed soil, pressure-sinkage bevameter testing is an effective way for assessing $k_{c}, k_{\phi}$ and $n$ values. Conversely, shear bevameter tests yield $\Phi$ values that, if used in SCM, would lead to significant overestimates of the real torque and drawbar pull force. 

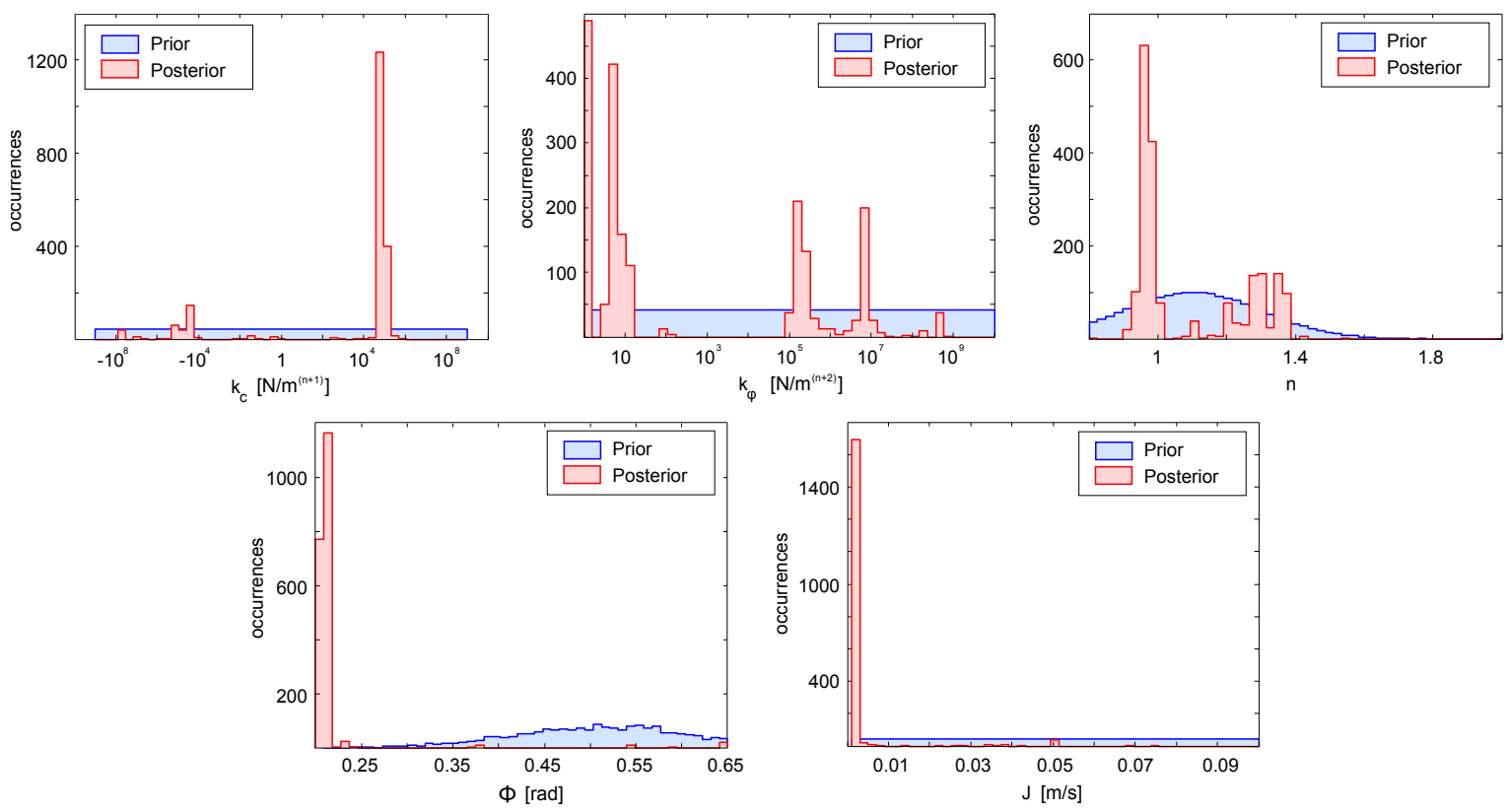

Figure 5: Univariate prior and posterior probability density functions.
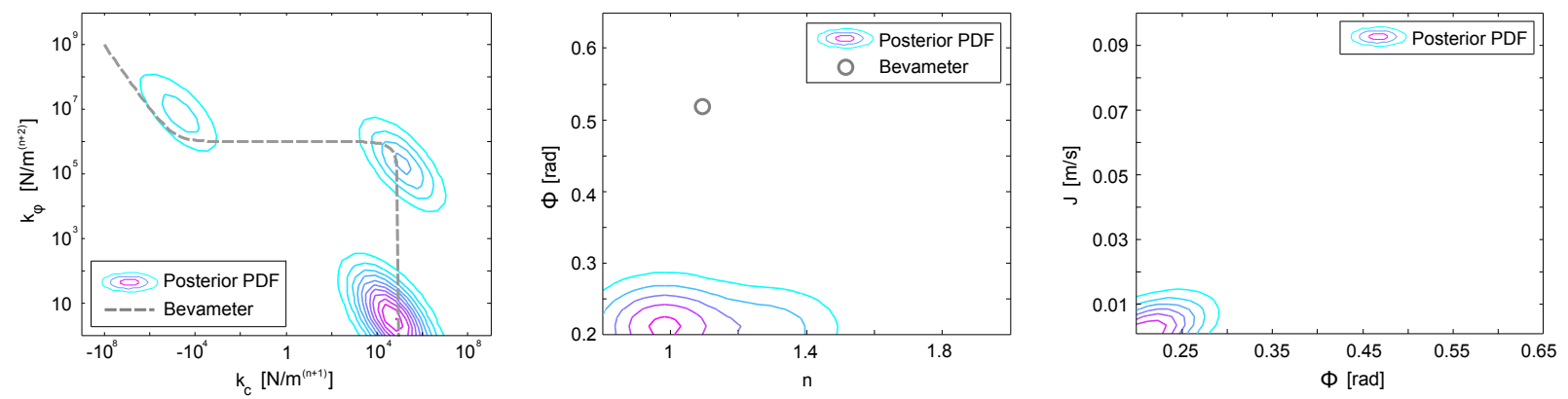

Figure 6: Bivariate posterior distributions of $k_{c}-k_{\phi}, n-\Phi$ and $\Phi-J$ pairs compared with prior information from bevameter measurements (mean values).

\subsection{Cases 2}

The aim of this second analysis is to study the influence of the wheel flexibility in the updating process and to assess the reliability of the parameter identification procedure. As in the previous subsection, the bivariate posterior distributions for the most interesting SCM parameter pairs are presented in Figure 7 (case 2a) and Figure 8 (case $2 \mathrm{~b}$ ) via contour plots and compared with bevameter measurements. In this case the outcomes can be summarized by the following statements:

- Both TMCMC simulations yield very close results. Yet, $k_{c}, k_{\phi}, n$ and $\Phi$ posterior PDFs appear very similar to those obtained in case 1. Either fact indicates a good level of reliability of the procedure.

- The posterior PDFs of $n$ appear narrower than in case 1 . This is most likely caused by the variability of the Janosi parameter removed in the second analysis.

- The flexibility of the wheel can be accounted for by increasing the wheel radius of the multi-body model. However, the level of importance of this parameter seems to be low. This assertion is motivated by the fact that many different $R$ values have a similar level of plausibility and that $R$ has no significant correlation with any of the analyzed parameters. 

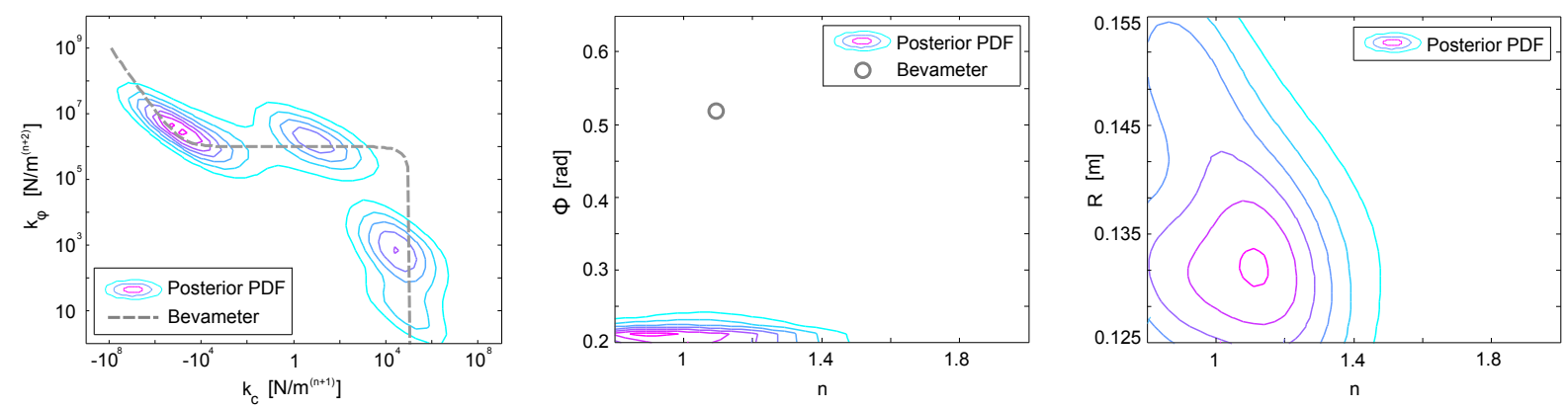

Figure 7: Bivariate posterior distributions of $k_{c^{-}} k_{\phi}, n-\Phi$ and $n-R$ pairs compared with prior information from bevameter measurements (mean values) for the case $2 \mathrm{a}$.
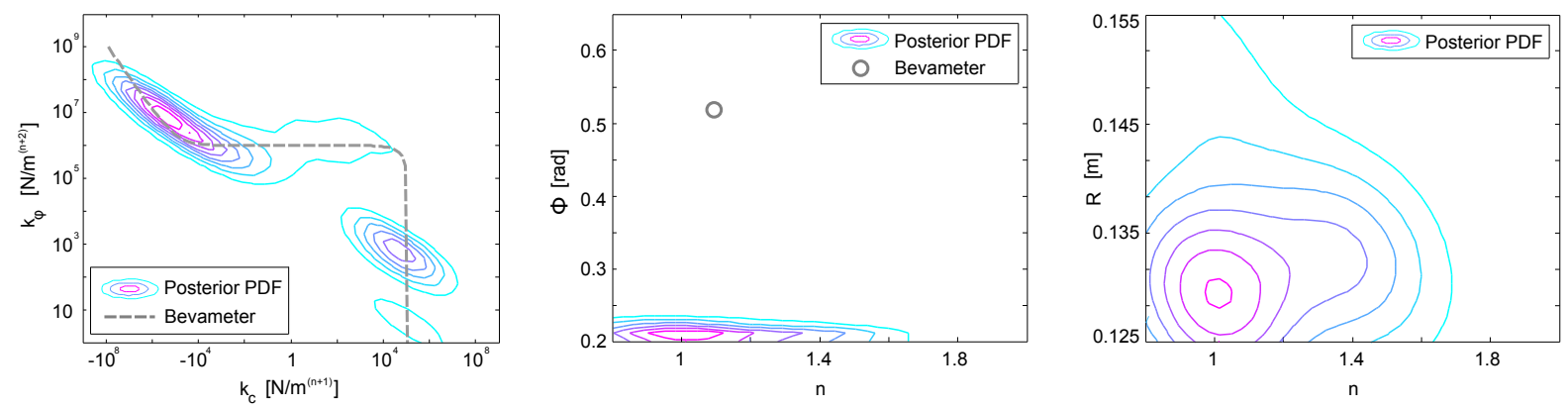

Figure 8: Bivariate posterior distributions of $k_{c}-k_{\phi}, n-\Phi$ and $n-R$ pairs compared with prior information from bevameter measurements (mean values) for the case $2 \mathrm{~b}$.

A comment can be made also about the accuracy of the posterior PDFs given by TMCMC. Although it has been proved that TMCMC yields a solution that converges asymptotically to the true solution (Ching and Chen, 2007), a very large number of samples may be sometimes needed to achieve exact posterior distributions. In this study the number of samples of each TMCMC simulations seems to suffice to solve the inference problem. However, the accuracy of the outcomes can be improved by enlarging the sample sizes. Indeed, since in the $k_{c}-k_{\phi}$ contour plots all points on the dashed lines produce identical contact forces, it is expected that an exact posterior distribution would follow such a trend that is only outlined by a single TMCMC solution. If the posterior sample points of the three TMCMCs are gathered in a unique set and the posterior distribution is evaluated, then the result appears much more accurate, as presented in Figure 9.

\subsection{General comments}

Contrary to deterministic model updating that yields parameter point estimates, the Bayesian approach identifies a set of highly plausible models. Posterior PDFs are much more informative than point estimates as they give a more comprehensive description of the model behavior by disclosing the existing correlation and localizing many different potentially good model parameter setups. The appropriateness of any model defined within the parameter space is measured by the corresponding posterior PDF value that represents the model plausibility level, which is the capability of the model to match experimental data and prior information. If this information is integrated into a decision theory framework, then the full power of the Bayesian approach can be used to take robust actions in condition of uncertainty.

The procedure is also a useful tool for model validation purposes. In this context, the study pointed out positive and critical aspects of SCM. On the one hand, the good agreement of the pressure-sinkage parameters identified by the procedure with those of bevameter tests proves the correct extension of the pressure-sinkage Bekker formula to tridimensional contact scenarios. On the other hand, substantial 


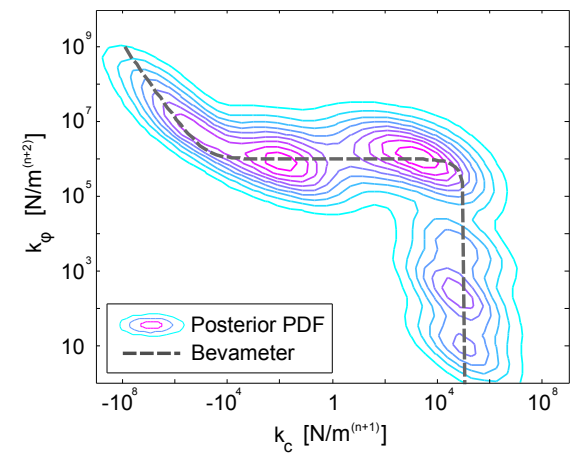

Figure 9: Bivariate $k_{c^{-}} k_{\phi}$ posterior distribution obtained by agglomerating the three TMCMC posterior sample sets.

mismatching in the friction angle warns the researcher against unreasoned use of the bevameter assessments in SCM. Interestingly, the same SCM behavior has also been observed in preceding analyses with different soils (Krenn and Hirzinger, 2008). Nevertheless, it is still unclear if the mismatching is due to modeling defects or to physical wheel-soil contact phenomena that cannot be caught by bevameter shear tests.

Another advantageous feature of Bayesian inference is its capability to efficiently handle data flows. Although calculation of posterior PDFs is generally challenging due to massive sampling, starting from a prior PDF shape not too different from the posterior one would require significantly smaller computational burden than a general case. For this reason, it is expected that Bayesian approach could be considered for on-board soil parameter estimations. Indeed, when solving close-in-time identification problems, in the absence of marked soil property discontinuities, successive posterior distributions should not be very different. This means that actual posterior PDFs could be determined with reduced computational effort. Significant computational speed-up can also be obtained by resorting to surrogate analytic models (Simpson et al., 2001) of the original Bekker-based contact models. This technique, already adopted in rover mobility prediction in condition of soil parameter uncertainty (Ishigami et al., 2009), is deemed to bring benefit also to the inference problem presented here.

\section{Conclusions}

Soil parameters of Bekker-based contact models for planetary rover mobility analysis are commonly estimated via bevameter tests. In this paper an alternative procedure is presented which makes use of both bevameter and single wheel experiments. The identification problem is addressed through a Bayesian procedure in which the initial guess on the soil parameters given by bevameter measurents is corrected by means of single wheel measurements. The analysis shows that, at least in the present case, bevameter measurements of the soil parameters can been reliably used in the contact model only to a certain extent. Major problems exist with regard to the friction angle for which the usage of bevameter measurements would lead to sensibly higher contact forces with respect to the values measured via single wheel tests. An advantage of Bayesian approach presented in the paper with respect to classical deterministic procedures is that it ensures a better treatment of uncertainties, which if integrated within a decision-making framework, may lead to robust actions in condition of uncertainty.

\section{References}

Apfelbeck, M., Kuß, S., Rebele, B., and Schäfer, B. (2011). A systematic approach to reliably characterize soils based on bevameter testing. Journal of Terramechanics, 48(5):360 - 371 . 
Beck, J. and Katafygiotis, L. (1998). Updating models and their uncertainties. part i: Bayesian statistical framework. Journal of Engineering Mechanics, 124:455-461.

Bekker, M. (1956). Theory of land locomotion. University of Michigan Press.

Bekker, M. (1969). Introduction to Terrain-Vehicle Systems. University of Michigan Press.

Bishop, C. (2006). Pattern recognition and machine learning. Springer, 1st ed. 2006. corr. 2nd printing edition.

Ching, J. and Chen, Y. (2007). Transitional markov chain monte carlo method for bayesian model updating, model class selection, and model averaging. Journal of Engineering Mechanics, 133(7):816-832.

Gallina, A., Krenn, R., Scharringhausen, M., and Schaefer, B. (2012). Bayesian updating of planetary rover wheel-soil contact model. In International Symposium on Artificial Intelligence, Robotics and Automation in Space (I-SAIRAS 2012), Turin, Italy.

Goller, B., Broggi, M., Clavi, A., and Schuëller, G. (2011). Efficient model updating of the goce satellite based on experimental modal data. In 3th International Conference on Computational Methods in Structural Dynamics and Earthquake Engineering (COMPDYN2011), Corfu, Greece.

Ishigami, G., Kewlani, G., and Iagnemma, K. (2009). Predictable mobility: A statistical approach for planetary surface exploration rovers in uncertain terrain. IEEE Robotics \&3 Automation Magazine, pages $61-70$.

Ishigami, G., Miwa, A., Nagatani, K., and Yoshida, K. (2007). Terramechanics-based model for steering maneuver of planetary exploration rovers on loose soil. Journal of Field Robotics, 24:233-250.

Janosi, Z. and Hanamoto, B. (1961). An analysis of the drawbar pull vs slip relationship for track laying vehicles. Report rr 47, Land Locomotion Laboratory.

Krenn, R. and Gibbesch, A. (2011). Soft soil contact modeling technique for multi-body system simulation. In Zavarise, G. and Wriggers, P., editors, Trends in Computational Contact Mechanics, volume 58 of Lecture Notes in Applied and Computational Mechanics, pages 135-155. Springer Berlin / Heidelberg.

Krenn, R. and Hirzinger, G. (2008). Simulation of rover locomotion on sandy terrain - modeling, verification and validation. In 10th ESA workshop on Advanced Space Techonolgies for Robotics and Automation (ASTRA), Noordwijk, Netherlands.

Leite, A., Gallina, A., and Schäfer, B. (2012). Parameter identificationand contact modeling for planetary wheeled prover in soft soil. In 16th IFAC Symposium on System Identification (SYSID 2012), Brussels, Belgium.

Metropolis, N., Rosenbluth, A., Rosenbluth, M., Teller, A., and Teller, E. (1953). Equations of state calculations by fast computing machines. Journal of Chemical Physics, 21(6):1087-1092.

Muto, M. (2007). Application of Stochastic Simulation Methods to System Identification. PhD thesis, California Institute of Techonology.

Saltelli, A., Chan, K., and Scott, E. (2000). Sensitivity Analysis. John Wiley and Sons.

Simpson, T., Poplinski, J., Koch, P. N., and Allen, J. (2001). Metamodels for computer-based engineering design: Survey and recommendations. Engineering with Computers, 17:129-150. 10.1007/PL00007198.

Wong (2010). Terramechanics and off-road vehicles engineering. Elsevier, 2nd ed. 2010 edition. 